\title{
Analysis of liver pathologies in stray dogs in the age aspect (sectional study)
}

\author{
L.V. Tkachenko* \\ FSBEI HE Altai SAU, Barnaul, Russian Federation
}

\begin{abstract}
The presence of stray animals in megacities or small settlements is the result of human activity. Stray dogs are in direct contact with representatives of wild fauna, influencing the occurrence and spread of various epizootics and epidemics. Stray animals, as a part of society, can serve as a biological model for understanding the influence of the environment on the body. We conducted a sectional study of 32 dogs aged from 2 years to 10 years and older. As a result, it was found that there is no correlation between sex and liver pathologies. Liver pathologies in stray animals were observed in the period from 2 to 7 years old, with a peak at 6 years of age, by 8 years old the number of pathologies decreases almost 2 3 times. Liver cirrhosis (45\%), protein dystrophy of the liver (45\%), acute congestive hyperemia of the liver (19\%), fatty liver dystrophy (16\%) and neoplasms in the liver $(13 \%)$ were recorded as often as possible in stray animals. The main causes of liver pathologies in stray animals were: stress, toxic effects of food or chemicals (poisoning), pathogens of infectious diseases, metabolic disorders, internal bleeding (car injury).
\end{abstract}

\section{Introduction}

Russian legislation defines stray animals as animals without the supervision of an owner, or animals without owners [1]. The presence of stray animals in megacities or small settlements is the result of human activity. Such animals always live near the human habitation, since there are always food niches [2,3]. In addition, stray dogs have direct contact with representatives of wild fauna [4,5]. All this directly or indirectly affects the occurrence and spread of various epizootics and epidemics, where stray dogs are carriers of pathogens [6].

At the same time, stray animals, as a part of society, can serve as a biological model for understanding the influence of the environment on the body.

The liver is the largest digestive gland in the body. Blood flows here through the portal vein, then through the hepatic capillaries "washes" the hepatocytes, participating in the neutralization of toxins, metabolic processes. That is, it participates in blood circulation and is a barrier between the gastrointestinal tract and other tissues. The liver is involved in the

\footnotetext{
* Corresponding author: rabota36@bk.ru
} 
secretion of bile into the duodenum, participating in digestion; it secretes hormones, thus being the gland of internal secretion [7].

We have described pathologies of the kidneys, pancreas, and gastrointestinal tract in homeless animals [8-10].

Thus, analyzing the pathological processes occurring in the liver in stray animals, it is possible to identify the influence of the environment on the body. To make predictions on the impact of negative environmental factors on the human body with a certain degree of interpretation.

\section{Materials and methods}

Studies were conducted on the corpses of 32 stray dogs (Table 1), outbred, physiologically mature [11], who died for various reasons (excluding euthanasia) in the period 2017-2020. The animals were supervised by animal protection organizations or volunteers of the Altai Krai.

The liver with a gallbladder was used as a material for research.

Table 1. Animals registered in the course of research

"Analysis of liver pathologies in stray dogs"

\begin{tabular}{|c|c|c|c|c|c|c|c|c|c|c|}
\hline \multirow[t]{2}{*}{ Sex } & \multicolumn{9}{|c|}{ Age, years old } & \multirow[t]{2}{*}{ Total } \\
\hline & 2 & 3 & 4 & 5 & 6 & 7 & 8 & 9 & $\begin{array}{l}10 \text { and } \\
\text { older }\end{array}$ & \\
\hline Male & 1 & 2 & 3 & 3 & 5 & 2 & 2 & - & 3 & 21 \\
\hline Female & 1 & 3 & 1 & 2 & 1 & 1 & 2 & - & - & 10 \\
\hline Total & & & & & & & & & & 31 \\
\hline
\end{tabular}

\section{Research methods}

1. Collection of anamnestic data (if available).

2. Registration of the animal in the journal "Results of pathoanatomical autopsy", indicating: sex; age [12]; breed; color; anamnesis; pathoanatomical diagnoses; cause of death.

3. Pathoanatomical autopsy according to the Shore method with a description according to the generally accepted scheme [12].

4. Photographing [13].

5. Statistical processing and analysis of the obtained data [14].

\section{Results and their discussion}

As a result of the analysis of the conducted studies, we noted that there is no correlation between sex and liver pathologies.

Figure 1 shows the registered changes in the liver in stray dogs. In one animal, several pathological changes were recorded in the organ under study. 


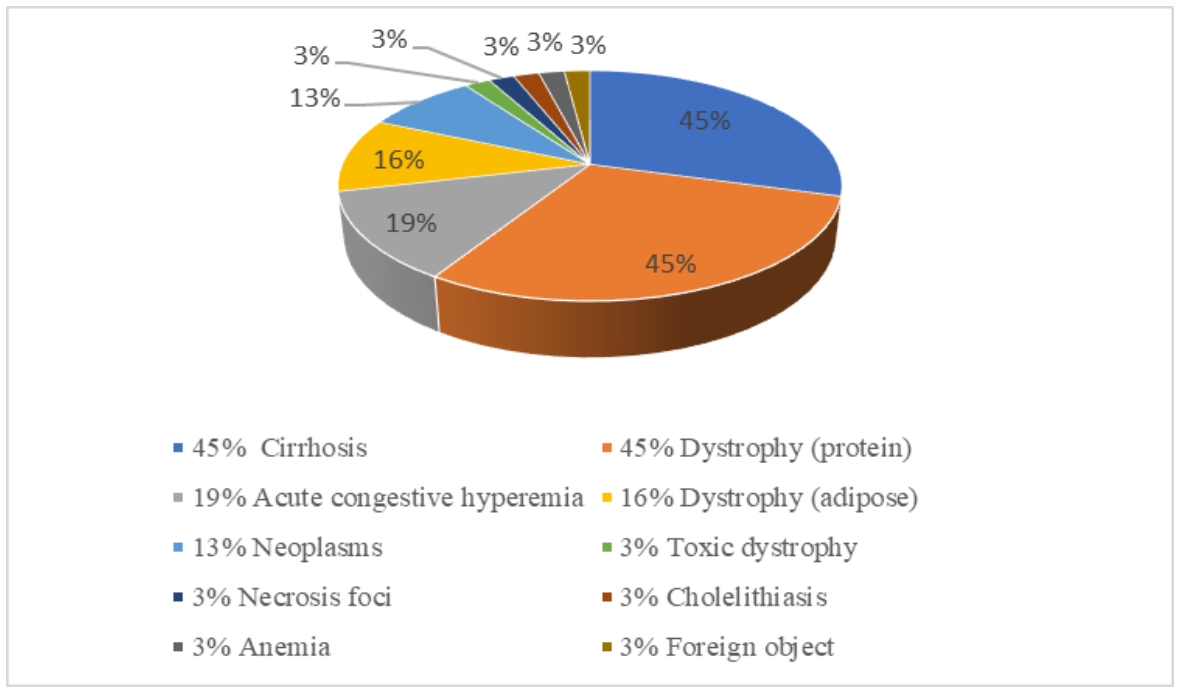

Fig. 1. Pathological changes in the liver in stray (in the period 201-2020)

Analyzing the results of the pathoanatomical autopsy and Figure 1, we conclude the following:

As often as possible $-45 \%$ of the studied cases in stray dogs account for liver cirrhosis (Fig. 2.A), it begins to manifest itself from the age of 3, reaching a peak by the age of 6 .

Cirrhosis is an irreversible diffuse liver lesion with fibrosis and changes in the architectonics of the vascular bed. In dogs aged 5 years and older, extensive cirrhotic liver lesions were observed. Based on anamnestic data, we believe that liver cirrhosis developed in these animals as a result of stress, toxic effects of feed and infectious disease pathogens.

In $45 \%$ of the studied animals, protein dystrophy of the liver was registered at the age of 3 to 8 years.

Protein liver dystrophy is a pathology that develops as a result of a violation of protein metabolism and deposition of hepatocytes in the cytoplasm, protein vascular wall. Analysis of anamnestic data showed that in these animals, liver protein dystrophy developed as a result of a violation of metabolic processes, as a way of life or as a consequence of exposure to infectious disease pathogens.

In $19 \%$ of the studied animals, acute congestive liver hyperemia was registered at the age of 4.5 and 7 years.

Acute congestive liver hyperemia is a circulatory disorder characterized by a violation of blood outflow with normal blood flow. Anamnestic data indicate that in these dogs this pathology was the result of the action of toxic substances of exogenous origin (poisoning).

In $16 \%$ of the studied animals, fatty liver dystrophy was registered at the age of 3 and 5 , 6 years (Fig. 2.B).

Fatty liver dystrophy is a pathology of metabolic processes, with the accumulation of lipoproteins in hepatocytes. Based on anamnestic data, we believe that in these animals, fatty liver dystrophy was the result of stress, toxic substances of exogenous origin and infectious disease pathogens.

In $13 \%$ of the studied animals, neoplasms of various origins were registered at the age of 3.4 and 6.7 years (Fig. 2.C).

It is not possible to explain the reasons for the development of neoplasms in the liver in the studied dogs.

In isolated cases, the following was in studied animals: 
$3 \%$ - toxic liver dystrophy at the age of 6 years, as a result of the action of toxic substances.

$3 \%$ - foci of necrosis at the age of 6 years, as a result of a parasitic disease.

$3 \%$ - cholelithiasis in gallbladder at the age of 2 years, as a result of metabolic disorders.
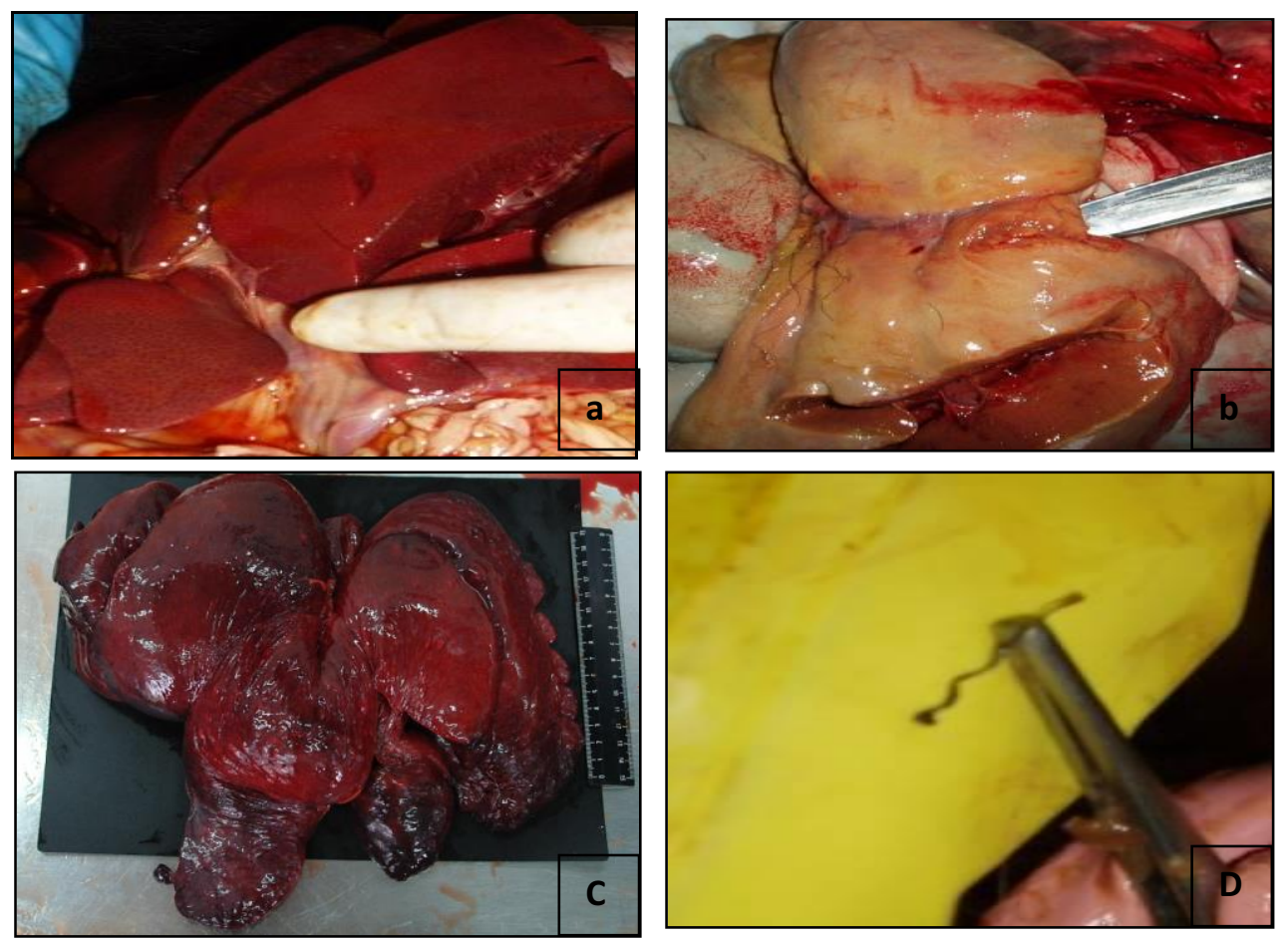

Fig. 2. Liver pathologies in stray dogs.

a. Liver cirrhosis. Male. Age 6 years. Outbred.

b. Adipose dystrophy. Female. Age 3 years. Outbred.

c. Neoplasm. Female. Age 6 years. Outbred.

d. Foreign object (wire). Male. Age 5 years. Outbred.

$3 \%$ - liver anemia at the age of 2 years, as a result of internal bleeding (car injury).

$3 \%$ - foreign object (wire) at the age of 5 years (Fig. 2.D), as a result of injury.

The percentage of liver pathologies by age is shown in Figure 2. 


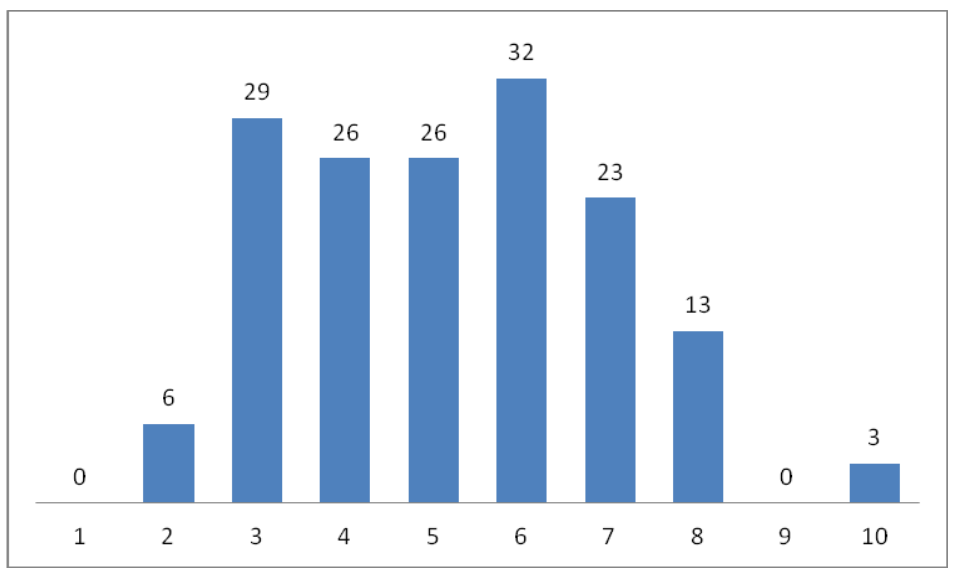

Fig. 3. The percentage of liver pathologies in stray dogs in the age aspect (where the upper figure is the $\%$ ratio to the common liver pathology, and the lower one is the age).

The analysis of Figure 3 showed that the number of pathological processes in the liver in the studied stray dogs increased sharply in the period from 3 to 7 years, with a peak at 6 years of age. By the age of 8 , the total number of liver pathologies decreases almost 2-3 times. Nevertheless, it should be remembered that this picture is typical only for the group of studied animals. We believe that this ratio can be partly explained by the largest number in the age groups, as well as outbreaks of infectious diseases and cases of mass poisoning. Violation of metabolic processes in stray animals is a consequence of lifestyle.

\section{Conclusions}

1. There was no correlation between sex and liver abnormalities in the studied dogs.

2. Liver pathologies in the studied stray dogs were observed in the period from 2 to 3 to 7 years, with a peak at 6 years of age. By the age of 8 , the total number of liver pathologies decreases almost 2-3 times.

3. Liver cirrhosis (45\%), protein dystrophy of the liver (45\%), acute congestive hyperemia of the liver (19\%), fatty liver dystrophy (16\%) and neoplasms in the liver (13\%) were recorded as often as possible in stray dogs under study.

4. The main causes of liver pathologies in the studied stray dogs were the following: stress, toxic effects of food or chemicals (poisoning), pathogens of infectious diseases, metabolic disorders.

\section{References}

1. Letter of the Ministry of Natural Resources and Ecology of the Russian Federation dated August 27, 2020, N 12-50/11056-OG "On the organization of activities in the treatment of animals without owners"

2. D.A. Indereykina, S.G. Kupriyanova, Young scientist, 1 (15), 90-91 (2018)

3. V. Thomas-Vaslin, Acta Biotheoretica, 68 (1), 171-199 (2020)

4. I.I. Rakhimov, E.Sh. Shamsuvaleeva, Bulletin of the Kazan State Pedagogical University, 3 (7), 142-152 (2006) 
5. N.N. Vinokurov, V.V. Rudoi, Acta Biologic Sibirica, 6 (5), 249-277 (2020)

6. Federal Law No. 498-FZ dated 27.12.2018 (as amended on 27.12.2019) "On Responsible Treatment of Animals and on Amendments to Certain Legislative Acts of the Russian Federation", Article 17

7. A.F. Klimov, A. I. Akaevsky, Anatomy of domestic animals (St. Petersburg: Lan, 462463, 2011)

8. L.V. Tkachenko, Bulletin of the Altai State Agrarian University, 4, 135-139 (2019).

9. L.V. Tkachenko, Bulletin of the Altai State Agrarian University, 8(178), 153-157 (2019)

10. L.V. Tkachenko, Bulletin of the Altai State Agrarian University, 12(194), 74-79 (2020)

11. A.P. Studentsov, V.S. Shipilov, V.Ya. Nikitin, Obstetrics, gynecology and biotechnics of animal reproduction (Saint-Petersburg: Lan, 548, 2020)

12. A.V. Zharov, Pathological anatomy of animals: textbook for universities (Saint Petersburg: Lan, 604, 2021) URL: https://e.lanbook.com/book/164712

13. P.N. Beschastnov, Fotographics (Moscow: RSU n.a. A.N. Kosygin, 61, 2010)

14. R.M. Gimazov, Statistical processing of research materials on a computer: an educational and methodological manual (Surgut: SurSPU, 138, 2015) 